\title{
Der Deutsche Juristinnen-Verein e.V.
}

Zwischen 1900 und 1909 wurden an den deutschen Universitäten Frauen an allen Fakultäten zum Studium zugelassen. Dies galt auch für Frauen an den juristischen Fakultäten. Während die meisten Frauen allerdings ihre Universitätsabschlüsse den männlichen Mitstudenten vergleichbar absolvieren konnten, war dies den Juristinnen verwehrt. Bis auf Bayern, wo sie das erste juristische Staatsexamen ablegen konnten, durften Frauen nicht an den Staatexamina sowie dem juristischen Vorbereitungsdienst teilnehmen. Sie beendeten ihr Studium mit dem Doktorexamen.

Die Ministerien, Universitäten und Vertreter der juristischen Berufe begründeten den Ausschluss der Frauen mit verschiedenen Normen aus dem Gerichtsverfassungsgesetz, der Rechtsanwaltsordnung, der Zivilprozessordnung sowie der Strafprozessordnung. Im Wesentlichen handelte es sich dabei um $\mathbb{S} 2$ des Gerichtsverfassungsgesetzes (GVG) vom 27. Januar 1877, später in neuer Fassung vom 1. Juni 1910, durch den die Befähigung zum Richteramt an das Bestehen der zwei juristischen Staatsexamina oder nach $\mathbb{4}$ an das Professorendasein an einer deutschen Universität gebunden war. Diese Normen waren zwar geschlechtsneutral formuliert, sie wurden aber geschlechtsspezifisch ausgelegt: Der Gesetzgeber habe Frauen in diesen Vorschriften nur deshalb nicht ausdrücklich ausgeschlossen, weil er die Möglichkeit, dass Frauen jemals diese Abschlüsse anstreben könnten, nicht in Betracht gezogen habe.

Die ersten promovierten deutschen Juristinnen konnten demnach in den klassischen juristischen Berufen nicht tätig werden und suchten ihr Auskommen in anderen Bereichen, die sich in der Regel im Kontext des sich neu formierenden Berufsfeldes der sozialen Arbeit fanden: Beratung in den Rechtsschutzstellen für Frauen, Lehr- und Führungstätigkeit an den sozialen Frauenschulen, Mitarbeit bei Rechtsanwälten, im Journalismus, im Staats- und Kommunaldienst, als wissenschaftliche Hilfsarbeiterin bei der Gewerbeinspektion und in der Jugendgerichtsfürsorge. ${ }^{1}$ Die Juristinnen beklagten, dass der Versuch, „die fehlende offizielle juristische Ausbildung zu ersetzen, eine Betätigungsmöglichkeit zu erreichen und sie sich zu erhalten, einen unverhältnismäßigen Aufwand an Mut und Kraft" erforderte, die allerdings „verbraucht werden, ehe es zu eigentlichen Berufsausübung kommt. “2 Sie wollten in den juristischen Berufen arbeiteten, für die sie studiert hatten.

Diese unbefriedigende Situation führte dazu, dass sich drei der frühen Juristinnen im Jahr 1914 zusammentaten, um eine erste akademische Berufsvereinigung zu gründen, den Deutschen JuristinnenVerein, e.V. (DJV). ${ }^{3}$ Bei diesen Gründerinnen handelte es sich um Dr. iur. Margarete Berent, Dr. iur. Margarete Muehsam-Edelheim, zu diesem Zeitpunkt noch Margarete Meseritz, und Dr. iur. Marie Munk. ${ }^{4}$ Sie riefen den Verein in Berlin ins Leben, um „die Interessen, insbesondere die beruflich wissenschaftliche Fortbildung der Juristinnen zu fördern “. 5 Der Verein war unabhängig, überparteilich und überkonfessionell. Im Vergleich mit anderen akademischen weiblichen Berufsgruppen wurde er 1914 sehr früh gegründet. Dies hing vermutlich damit zusammen, dass die anderen Akademikerinnen bessere Berufsvoraussetzungen hatten, so dass die Notwendigkeit der Gründung einer Berufsvereinigung nicht so früh bestand wie bei den

1 Bernhard, Margarete, Vorbildungs- und Berufsverhältnisse der deutschen Nationalökonominnen und Juristinnen, in: Volkswirtschaftliche Blätter, XII. Jg. (1913), Nr. 5/6, S. 54-59 (57); Langsdorff, Erna von, Die Berufsaussichten der Juristin in Deutschland, in: Die Frau 20 (1912/13), S. 603-609 (603).

2 Langsdorff, ebd., S. 603; Berent, Margarete, Die Zulassung von Frauen zu den juristischen Berufen, in: Die Frau 27 (1919/20), S. 332-334 (332).

3 Früher wurden nur 1890 der „Allgemeine Deutsche Lehrerinnenverein“ und 1908 der „Verband akademisch gebildeter und studierender Lehrerinnen" gegründet, der sich 1925 in den „Deutschen Philologinnenverband“ umbenannte. Der „Bund deutscher Ärztinnen“ gründete sich erst 1924, als es schon fast 1400 Ärztinnen gab. Vgl. Huerkamp, Claudia, Bildungsbürgerinnen. Frauen im Studium und in akademischen Berufen 1900-1945, Göttingen 1996, S. 206 ff., 249 ff.

4 Zu ihren Biografien siehe: Röwekamp, Marion, Juristinnen. Lexikon zu Leben und Werk, hg. vom Deutschen Juristinnenbund, Baden-Baden 2005, S. 36-40, S. 271-274, S. 275-279.

5 Das genaue Gründungsdatum lässt sich nicht mehr feststellen, weil die Akten des Vereinsregisters Berlin den Folgen des Zweiten Weltkrieges zum Opfer gefallen sind und auch im Geheimen Preußischen Staatsarchiv unter den Juristenvereinigungen keine Materialen zum Deutschen Juristinnenverein e.V. zu finden sind. Vgl. auch: Juristinnenbund, Juristinnen in Deutschland, S. 5. Begrenzen lässt sich das Datum nur dahingehend, dass der Weltkrieg nach Berichten von Marie Munk schon ausgebrochen war: Marie Munk, Marie Munk, Autobiographie, LA Berlin, B Rep. 235-12, Autobiographie, S. IX, 4.

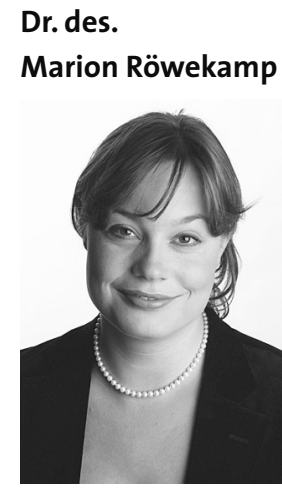

Ass. jur., Mexiko-City 
Juristinnen. Der Bund deutscher Ärztinnen zum Beispiel gründete sich erst zehn Jahre später, obwohl diese Berufsgruppe ungleich größer war. ${ }^{6}$ Der DJV blieb dagegen immer klein. In seinem Gründungsjahr hatte er 28 Mitglieder, 1916/17 35 Mitglieder ${ }^{7}$ und 191985 ordentliche sowie einige außerordentliche Mitglieder. ${ }^{8} 1927$ gab es 100 Mitglieder, bis 1932 stagnierte die Mitgliederzahl. ${ }^{9}$ Im August 1919 bestand der Vorstand des DJV aus sieben Juristinnen: Margarete Muehsam-Edelheim als der ersten Vorsitzenden, Marie Munk als der zweiten, Margarete Berent als Schatzmeisterin, Alice Eisner als Schriftführerin sowie Elsa Duhne, Elisabeth Hamburger-Schmitt und Maria Otto als einfache Vorstandsmitglieder. Mitte 1920 wechselte der erste Vorsitz zu Margarete Berent, die ihn bis März 1933 innehielt. Zweite Vorsitzende blieb Marie Munk, Schatzmeisterin wurde ein Frl. Hagemeyer, zu vermuten ist, dass es sich um die spätere Richterin Maria Hagemeyer handelt, ab 1928 übernahm die Assessorin Doris Pfeiffer das Amt. ${ }^{10}$ Die Geschäftsstelle führte seit Gründung des Vereins bis mindestens 1933 Lilli Seligsohn. Ab dem Jahr 1928 war eine erste Ortsgruppe des DJV in Hamburg unter der Führung von Mathilde Möller-Bing verzeichnet. ${ }^{11} 1933$ übernahm Dr. Ilse Adams den Vorsitz des DJV, Charlotte von Einem war Schriftführerin. ${ }^{12}$

Der DJV war so klein, dass er sich um die Interessen einzelner Sondergruppen innerhalb des Vereins kümmern konnte. Gerade wegen seiner geringen Mitgliederzahlen besaß er eine klare Übersicht darüber, wer wo arbeitete und wie es den Mitgliedern wirtschaftlich erging. Er veranstaltete regelmäßige Umfragen zur wirtschaftlichen und beruflichen Situation seiner Mitglieder; zum Beispiel 1917 eine Umfrage über die Kriegstätigkeit der deutschen Juristinnen. Alle diese Umfragen ergaben aber, wie Margarete Berent schrieb, selbst wenn sie vollständig beantwortet wurden, immer nur ein Augenblicksbild, von dem wiederum nur zwei Fälle für die Nachwelt erhalten geblieben sind. ${ }^{13}$ Der Verein veröffentlichte eine Form von Rundbrief, der die Mitglieder über die Tätigkeiten auf dem Laufenden hielt. ${ }^{14}$ Wie aus einem Zeitungsartikel hervorgeht, tagte der DJV in einer Generalversammlung. ${ }^{15}$

So klein der Verein mitgliedertechnisch auch gewesen sein mochte, war er außerordentlich aktiv für sein eigenes Anliegen, die Förderung der Situation der Juristinnen. Alle bekannten Petitionen an den Reichstag, die Länderparlamente sowie das Reichsjustizministerium und die Landesjustizverwaltungen des DJV beschäftigten sich in den ersten Jahren ausschließlich damit, den Zugang zu den juristischen Berufen zu erlangen. „Und dieser Kampf ist umso quälender“, schrieb eine Juristin, „je stärker das juristische Interesse ist, und je lebendiger das Gefühl dafür ist, dass die weiblichen Juristen neben den allgemeinen noch besondere Aufgaben in der Rechtspflege zu erfüllen haben; denn um so weniger wird die Betreffende sich darauf einstellen können, dauernd eine Tätigkeit ausüben zu können, bei der der juristische Teil unwesentlicher ist, um so schwerer wird es ihr werden, auf die spezifische Berufsausübung zu verzichten. Und je stärker ihr Gerechtigkeitsgefühl entwickelt ist, um so mehr wird es sich gegen die Ungerechtigkeit empören, ihr, nicht wegen mangelhafter Leistungen, sondern ihres Geschlechts wegen die Bahn zu verschließen. “16 Sehr hilfreich war, dass die erste Vorsitzende des Vereins, Margarete Muehsam-Edelheim, die Frauenbeilage der progressiven „Vossischen Zeitung“ leitete, was dem Verein zu einer exzellenten Öffentlichkeitswirkung verhalf. Alle wesentlichen Schritte auf dem Weg zur Öffnung der juristischen Berufe für Frauen wurden von der Berichterstattung der Vossischen Zeitung erfasst. Auch viele der einzelnen DJVMitglieder waren aktiv, schrieben Artikel für Fachzeitungen, Frauenblätter oder öffentliche Medien ${ }^{17}$, hielten Vorträge und nahmen an Podiumsdiskussionen teil, um ihre Situation in der Öffentlichkeit bekannt zu machen. ${ }^{18}$ Die Mitglieder des DJV nutzten dabei ihre persönlichen Verbindungen, die ihnen aufgrund ihrer bildungsbürgerlichen Herkunft zur Verfügung standen und Zugang zu Positionen und Persönlichkeiten erlaubten, die nicht-akademische Berufsgruppen in dieser Art nicht wahrnehmen konnten. Sie verhalfen ihnen zu persön-

61933 gab es bspw. bereits 3376 Ärztinnen von insgesamt 51.527 Ärzten, vgl. Grossmann, Atina, New Women in Exile: German Women Doctors and the Emigration, in: Ouack, Sybille ( $\mathrm{Hg}$.), Between Sorrow and Strength. Women Refugees of the Nazi Period, Cambridge 1996, S. 215-238 (219).

7 Jahrbuch des Bundes Deutscher Frauenvereine 4 (1915). Kriegsjahrbuch des Bundes Deutscher Frauenvereine, Mannheim/Berlin/Leipzig 1915, S. 102.

8 Margarete Berent-Collection, LBI NYC; das Jahrbuch des Bundes Deutscher Frauenvereine 8 (1919), Mannheim/Berlin/Leipzig 1919, S. 96, vermerkt für 1919 dagegen 79 Mitglieder.

9 Jahrbuch der Berufsverbände im Deutschen Reich, Ausgabe 1927, S. $136 ; 1927 / 27$, S.12; 1928-32, S. 11.

10 Jahrbuch des Bundes Deutscher Frauenvereine 10 (1921-1927), Adressverzeichnis, Mannheim/Berlin/Leipzig 1927, S. 12; Jahrbuch des Bundes deutscher Frauenvereine 12 (1928-1932), Mannheim/ Berlin/Leipzig 1932, Adressverzeichnis, S. 11.

11 Jahrbuch des Bundes Deutscher Frauenvereine 12 (1928-1932), aaO., Adressverzeichnis, S. 11.

12 Vgl. Schreiben des DJV vom 29.4.1933 an Margarete Berent, Margarete Berent Collection, LBI NYC.

13 Vgl. Berent, Margarete, Die Frau in den juristischen Berufen, in: Die Frau der Gegenwart 11 (1917), S. 153-157 (155).

14 Rundschreiben des DJV von Februar 1921:, Sehr geehrte Kommilitonin! [...] Deutscher Juristinnen Verein, Entwurf, Berlin, im Februar 1921“, Margarete Berent Collection, LBI NYC.

15 Edelheim, Margarete, Fräulein Richter und Frl. Rechtsanwalt, zwei Jahre Volljuristinnen, in: Vossische Zeitung vom 20.6.1926, o.S.

16 Berent, Margarete, Die Zulassung von Frauen zu den juristischen Berufen, in: Die Frau 27 (1919/20), S. 332-334 (333).

17 Z.B. Meseritz, Margarete, Das Recht der Frau, in: Berliner Allgemeine vom 27.3.1918, o.S.; dies., Die Frau in den akademischen Beruf, in: Vossische Zeitung vom 9.11.1919, o.S.; dies., Fräulein Referendar. Neue Rechte der Beamtinnen, in: Vossische Zeitung vom 25.1.1921, o.S.; Munk, Marie, Die Juristin. Merkblätter für Berufsberatung. Deutsche Zentralstelle für Berufsberatung der Akademiker, Berlin 1928; dies., Die juristische Ausbildung der Frauen, in: DJZ 30 (1925), S. 281 f.; dies., Ist die Frau für den Richterberuf befähigt? in: Tages-Fragen, Arbeiten erster Autoren für Tageszeitungen, hg. R. R. Daammert, Berlin 1927; dies., Sind weibliche Schöffen und Geschworene, Richter und Anwälte notwendig und wünschenswert? in: Staatsanzeiger, 13.5.1926, o.S.; Berent, Margarete: Die Frau als Richter, in: JW 49 (1920), S. 1012; dies., Die Frau in den juristischen Berufen, in: Die Frau der Gegenwart (1917), S. 153-157; dies., Die Zulassung von Frauen zu den juristischen Berufen, in: Die Frau 27 (1919/20), S. 332-334; dies., Weibliche Schöffen, in: Die Verhandlungen des fünften deutschen Jugendgerichtstages in Jena 1920, S. 54-58; Hegemann-Springer, Edith, Die Zulassung von Frauen zum juristischen Vorbereitungsdienst, in: Leipziger Zeitschrift für Deutsches Recht 1919, S. 1067-1069. 
lichen Gesprächen mit den betreffenden Justizministern und den Abgeordneten der einzelnen Fraktionen der Landtage und des Reichstags, um diese für ihr Anliegen zu sensibilisieren. Alle drei Gründerinnen des DJV waren Mitglied der neu gegründeten Deutschen Demokratischen Partei, die in den Folgejahren häufig als Sprachrohr des DJV im Reichstag fungierte. Eine spezifische Beschreibung der Forderungen und der Taktiken des DJV im Kampf um die Zulassung zu den juristischen Berufen führt in diesem Rahmen allerdings zu weit.

Es sind so wenige Informationen über den DJV erhalten, dass nur vorsichtige Rückschlüsse auf seine Tätigkeitsgebiete neben der Förderung der eigenen Berufsziele gezogen werden können. Es deutet sich allerdings an, dass der DJV für den Bund deutscher Frauenvereine (BDF), dem er wohl im Jahr 1916 im Gesamten beitrat, als eine Art „Rechtsabteilung“ fungierte. Petitionen des BDF, so zum Beispiel im Fall der Petition zur Reform des Strafrechtes und der Strafprozessordnung aus dem Jahr 1919, waren vom DJV im Auftrage des BDF zu prüfen und eine Empfehlung zum Vorgehen auszusprechen. ${ }^{19}$ Der Verein äußerte sich aber auch außerhalb der Tätigkeit seiner einzelnen Mitglieder und seiner rechtlichen Beratungstätigkeit für den BDF eigenständig zu Themen wie die Veränderung des Familienrechts sowie die Besserstellung der weiblichen Beamten. Insbesondere familienrechtliche Fragen schienen bei den Treffen des DJV regelmäßig Thema gewesen zu sein, wie den spärlich erhaltenen Erinnerungen der Mitglieder zu entnehmen ist. ${ }^{20}$ Der Verein engagierte sich auch im Rahmen der Doppelverdienerkampagne und der Neuregelung der Rechte weiblicher Beamter. Während sich viele Beamtenverbände wie der Deutsche Beamtenbund und der Verein der Reichspost- und Telegrafenbeamtinnen aber auch der BDF im Rahmen der Doppelverdienerkampagne für das freiwillige Ausscheiden von Beamtinnen mit einer angemessenen Abfindungssumme einsetzten, ${ }^{21}$ stand der DJV auf der Seite des Allgemeinen deutschen Lehrerinnenvereins, der das freiwillige Ausscheiden von Beamtinnen nicht akzeptierte und für die Gleichstellung männlicher und weiblicher Beamter eintrat. ${ }^{22}$ Er forderte gemeinsam mit dem Deutschen Philologinnenverband, der Vereinigung der Nationalökonominnen Deutschlands und dem Deutschen Verband der Sozialbeamtinnen die strikte Wiederherstellung des Art. 128 II WRV. ${ }^{23}$

Das Engagement innerhalb des DJV hatte sich für die Generation, die noch nicht an den Staatsexamina teilnehmen durfte, angesichts der fehlenden Berufsaussichten wie von selbst ergeben. Die Juristinnen empfanden den Beitritt wohl weniger als einen „radikalen“ Entschluss zum Beitritt zur bürgerlichen Frauenbewegung, sondern als konsequente Ergänzung zu ihrem Studium. Nicht zwingend waren die Juristinnen schon innerhalb der Familie durch ihre Mütter für Frauenfragen sensibilisiert worden, obwohl das durchaus vorkam. Entweder dahingehend, dass die Mütter bereits Mitglieder der Frauenbewegung waren oder mit ihr sympathisierten oder im Gegenteil, dass die Töchter das Schicksal ihrer Mütter nicht teilen wollten. Marie Munk stammte beispielsweise aus einer Familie, in der über die Frauenfrage nicht gesprochen wurde.
Sie gewann ihr Interesse daran erst durch ihre Arbeit für die Frauenrechtsschutzstelle in München. Die Einblicke in die beschränkten Rechte der Ehefrau gegenüber dem Ehemann machten ihr deutlich, dass es hier einer Rechtsreform bedurfte, zu der sie als Juristin auf eine besondere Art beitragen konnte. ${ }^{24}$ Indem also die deutsche bürgerliche Frauenbewegung den frühen Juristinnen in den Rechtsschutzstellen Arbeitsmöglichkeiten bot, erfuhren die Juristinnen hautnah von den rechtlichen Schwierigkeiten, mit denen die Frauen im Allgemeinen zu kämpfen hatten. Das stärkte wiederum die Sensibilität der Juristinnen für die Fragen der Frauenbewegung. Hinzu kamen die eigenen beschränkten Möglichkeiten, einen juristischen Beruf auszuüben. Der nahezu selbstverständliche Beitritt einer Jurastudentin zum Deutschen Juristinnen-Verein zeigt sich auch an der hohen Zahl der Juristinnen, die dem Verein bis 1922 bereits beigetreten waren. Von den 126 Juristinnen, die bis 1922 ihr Studium mit der Promotion abgeschlossen hatten, waren 47 Mitglied des DJV.

Während sich bis zum Erlass des „Gesetzes über die Zulassung der Frauen zu den Ämtern und Berufen der Rechtspflege“ im Jahr 1922 in den Archiven zahlreiche Eingaben und Petitionen des DJV finden, gibt es nach 1922 nur noch sehr vereinzelt Hinweise auf das Wirken des Vereins. 1926, vier Jahre nach der Zulassung zu den Berufen der Rechtspflege, beschäftigte sich der DJV auf seiner Generalversammlung mit den Berufsaussichten der Juristinnen. Die Vorsitzende Marga-

18 Vgl. z.B. Vortrag von Margarete Berent, „Die Frau in der Rechtspflege“, anlässlich der 15. Generalversammlung des BDF in Eisenach, Vortrag in der Margarete Berent Collection, LBI NYC; dies., Die Frau als Richter. (Vortrag von Dr. Margarete Berent auf der Versammlung der Politischen Arbeitsgemeinschaft der Frauen von Groß-Berlin am 6.12.1920, in: JW 49 (1920), S. 1012; Anna Mayer bei der Reichsfrauentagung der deutschen Volkspartei auf dem Stuttgarter Parteitag, vgl. Anonym, Die Schranken fallen. Die Frauen der Volkspartei für die Juristin, in: Neue Justiz vom 18.12.1921, o.S.; Margarete Berent bei einer Beratung der forensisch-medizinischen Vereinigung im Januar 1922, vgl. Muehsam, Die Eignung der Frau zum Richteramt. Mediziner und Juristen über das Problem, in: Vossische Zeitung, 22.1.1922, o.S.

19 Vgl. Brief von Marie Munk an Alice Bensheimer, 11.7.1919, LAB B Rep. 235-01 Fiche 2150.

20 So Elsa Koffka in Entschädigungsakte Marie Munk, Landesverwaltungsamt Berlin, Nr. 60.798.

21 Schütz, Dieter, Zwischen Standesbewusstsein und gewerkschaftlicher Orientierung. Beamte und ihre Interessenverbände in der Weimarer Republik, Baden-Baden 1992, S. 100; Nienhaus, Ursula; Vater Staat und seine Gehilfinnen - Die Politik mit der Frauenarbeit bei der deutschen Post (1864 - 1945), Frankfurt/Main, New York 1995, S. 15 ff.; Twellmann-Schepp, Margrit, Die Deutsche Frauenbewegung. Ihre Anfänge und erste Entwicklung 1843-1889, Meisenheim 1972, S. $98 \mathrm{f}$.

22 Hahn, Claudia, Der öffentliche Dienst und die Frauen - Beamtinnen in der Weimarer Republik, in: Frauengruppe Faschismusforschung (Hg.), Mutterkreuz und Arbeitsbuch. Zur Geschichte der Frauen in der Weimarer Republik und im Nationalsozialismus, Frankfurt/M. 1981, S. 49-78 (73), Kampmann, Doris, „Zölibat - ohne uns!“ - Die soziale Situation und politische Einstellung der Lehrerinnen in der Weimarer Republik, in: Frauengruppe Faschismusforschung $(\mathrm{Hg}$.$) ,$ Mutterkreuz und Arbeitsbuch. Zur Geschichte der Frauen in der Weimarer Republik und im Nationalsozialismus, Frankfurt/M. 1981, S. 79-104 (85); Huerkamp, Bildungsbürgerinnen, aaO., S. 217.

23 Schreiben dieser Verbände vom 26.4.1931 an alle Ministerien, BA Berlin R 431/2556.

24 Marie Munk, Autobiographie, LA Berlin, B Rep. 235-12, Autobiographie, S. IX, 3. 
rete Berent hatte eine Übersicht über die Lage und die Berufsaussichten der Juristinnen gegeben, die durch Berichte von Assessorinnen, Referendarinnen, Studentinnen und Vertreterinnen anderer Berufsgruppen ergänzt wurden. ${ }^{25}$ Zwei Jahre später findet sich eine Notiz, dass bei der Gründung einer Internationalen Vereinigung von weiblichen Rechtsanwälten in Paris im Juli 1928 als deutsche Vertreterin die Rechtsanwältin Margarete Berent anwesend war. ${ }^{26} 1933$ wandte sich die Schriftführerin des DJV, Lilli Seligsohn, an das preußische Justizministerium, um statistisches Material für Zwecke der Berufsberatung und zur Teilnahme an einer im Frühjahr in Berlin stattfindenden Ausstellung „Die Frau” zu erhalten. Der DJV versandte deshalb einen Fragebogen an alle deutschen Juristinnen, deren Anschriften er neben denen der eigenen Mitglieder ermitteln konnte. ${ }^{27}$

Was mit dem Deutschen Juristinnen-Verein 1933 geschah, ist unklar. Gesichert ist, dass er Ende April 1933 noch bestand $^{28}$, aber im Verlauf der Zeit wohl aufgelöst wurde, um der „Gleichschaltung“ zu entgehen. ${ }^{29}$ Viele seiner Mitglieder mussten emigrieren, einige wurden ermordet wie Cäcilie Holländer, geb. Jacobi, die am 14. November 1941 von Berlin nach Minsk deportiert wurde. Wieviele Mitglieder des DJV nach 1949 noch in Deutschland lebten oder zurückkehrten ist nicht bekannt. Jedenfalls vier aus der Mitgliederliste des DJV von 1919, Dr. Else Heinze-Piorkowski, Dr. Aenne KurowskiSchmitz, Dr. Maria Otto und Alice Pfahl, sind dem 1948 neu gegründeten Juristinnenbund beigetreten.

25 Edelheim, Margarete, Fräulein Richter und Frl. Rechtsanwalt, zwei Jahre Volljuristinnen, in: Vossische Zeitung, 20.6.1926, o.S.

26 Meldung im Berliner Tageblatt vom 22.7.1928, o.S., in: BA R 3001/4182, S. 75 .

27 Deutscher Juristinnenverein, Berlin, den 2.2.1933, an das Preußische Justizministerium., GStA Rep. 84 a, Fiche 12989, Bl. 219.

28 Dankesschreiben des Deutschen Juristinnen-Vereins an Margarete Berent am 29.4.1933 für ihre Arbeit. Margarete Berent Collection, LBI NYC.

29 So Zahn-Harnack, Agnes, Geschichte des deutschen Akademikerinnenbundes, 1926-1933, in: dies., Schriften und Reden 1914-1950, Tübingen 1964, S. 1-8 (7); vgl. auch Huerkamp, Bildungsbürgerinnen, S. 287.

\section{Juristinnen 1933 bis 1949 - unter besonderer Berücksichtigung der Situation jüdischer Frauen}

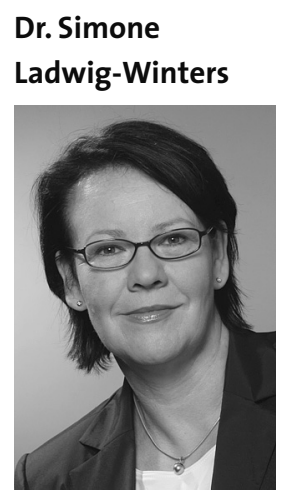

Historikerin, Berlin
Margarete Berent, 1887 in Berlin geboren, wartete lange darauf, die formale Qualifikation einer Volljuristin zu erlangen. ${ }^{1}$ Trotz Studiums und einer Dissertation $^{2}$, die 1913 so viel Anerkennung gefunden hatte, dass Otto von Gierke sie in seiner angesehenen Schriftenreihe veröffentlichte, wurde ihr die Zulassung zum Staatsexamen verweigert. Sie übernahm juristische Hilfsarbeiter- und Wohlfahrtsberaterstellen, war während des Krieges Dezernentin im juristischen Büro der AEG, bis sie 1917 erst beim Zweckverband Groß-Berlin, dann in der Stadtverwaltung Groß-Berlins angestellt wurde. 1919, sobald sich die Möglichkeit in Preußen bot, legte sie dann endlich ihr erstes Staatsexamen ab. Doch der Vorbereitungsdienst blieb ihr vorerst weiterhin verschlossen. Sobald sich hier die Möglichkeit bot, 1922, meldete sie sich dazu und bestand 1925 die große Staatsprüfung. Im gleichen Jahr ließ sie sich im Alter von 38 Jahren als Rechtsanwältin in Berlin nieder.

Margarete Berent ist ein Beispiel dafür, dass sich mit der Zulassung von Frauen zum Staatsexamen zu Beginn der Weimarer Zeit ganz neue Perspektiven eröffneten. Nun konnten Frauen dauerhaft als Juristinnen arbeiten. Damit erweiterte sich das Spektrum der möglichen Berufe, und sie konnten sich nach der Assessorprüfung als Anwältinnen niederlassen oder das Amt einer Richterin oder gar Staatsanwältin übernehmen.
Eine der wenigen, denen es gelang, in den Staatsdienst zu kommen, war Marie Munk (geb. 1885). ${ }^{3}$ Auch sie hatte Umwege machen müssen, um Volljuristin zu werden. Dass sie eine sehr gute Juristin war, erkannten auch die öffentlichen Stellen. Nach einer fünfjährigen Tätigkeit als Anwältin bewarb sie sich für das Amt einer Richterin und wurde tatsächlich 1930 zur ersten Landgerichtsrätin in Preußen ernannt.

Marie Munk kam, wie Margarete Berent oder auch Hanna Katz, aus einer jüdischen Familie. Gerade die jüdischen Familien waren aufgeschlossener, Töchter den Weg in den so männlich dominierten Bereich beschreiten zu lassen. Die Nähe zum Recht war durch die Religion, in der schon immer über Streitfragen diskutiert wurde, vertraut.

1 Zum Leben Berents, die auch eine der Mitbegründerinnen des Juristinnen-Vereins e.V. gewesen ist, s. Ladwig-Winters, Simone: Anwalt ohne Recht - Das Schicksal jüdischer Rechtsanwälte in Berlin nach 1933. 2. Aufl. Berlin 2007; S. 120; Röwekamp, Marion: Juristinnen Lexikon zu Leben und Werk, hrsg. vom Deutschen Juristinnenbund e.V., Baden-Baden 2005, S. 36-40.

2 Berent, Margarete: Die Zugewinnstgemeinschaft der Ehegatten. Breslau 1915, in der Reihe „Untersuchungen zur deutschen Staats- und Rechtsgeschichte, Heft 123; diese Dissertation fand dann viele Jahre später, 1959, bei der Eherechtsreform der Bundesrepublik Deutschland Berücksichtigung.

3 Personalakte, s. BArch R 3001, 69.257; Röwekamp (2005), S. 275-279. 